\title{
Surface States Effect on the Large Photoluminescence Redshift in GaN Nanostructures
}

\author{
Ahmed Ben Slimane ${ }^{1}$, Adel Najar ${ }^{1}$, Tien Khee $\mathrm{Ng}^{1}$, Damián P. San-Román-Alerigi ${ }^{1}$, Dalaver Anjum ${ }^{2}$, Boon $\mathrm{S}$ \\ Ooi ${ }^{1 *}$ \\ ${ }^{\text {I} P h o t o n i c s ~ L a b o r a t o r y, ~ K i n g ~ A b d u l l a h ~ U n i v e r s i t y ~ o f ~ S c i e n c e ~ a n d ~ T e c h n o l o g y ~(K A U S T), ~ T h u w a l ~ 23955-6900, ~ S a u d i ~ A r a b i a . ~}$ \\ ${ }^{2}$ Advanced Nanofabrication and Imaging Core-Lab, King Abdullah University of Science and Technology (KAUST), Thuwal 23955-6900, Saudi \\ Arabia \\ *corresponding author: boon.ooi@kaust.edu.sa
}

\begin{abstract}
We report on the large photoluminescence redshift observed in nanostructures fabricated using n-type GaN by ultraviolet (UV) metal-assisted electroless chemical-etching method. The scanning electron microscopy (SEM) characterization showed nanostructures with size dispersion ranging from 10 to $100 \mathrm{~nm}$. We observed the crystalline structure using high resolution transmission electron microscopy (HRTEM) and electron energy loss (EELS) techniques. In contrast to $362 \mathrm{~nm}$ UV emission from the GaN epitaxy, the nanostructures emitted violet visible-light in photoluminescence (PL) characterization with increasing optical excitation. An energy band model was presented to shed light on the large PL redshift under the influence of surface states, which resulted in two competing photoluminescence mechanisms depending on excitation conditions.
\end{abstract}

OCIS codes: (160.4236) Nanomaterials; (160.6000) Semiconductor materials; (250.5230) Photoluminescence; (300.6490) Spectroscopy, surface

\section{Introduction}

III-Nitride materials are of great interest for solid state lighting applications because of their wide band-gap tunability from UV to visible range [1,2]. The emission of the bulk GaN usually occur at the ultra violet (UV) photon energy of $\sim 3.42 \mathrm{eV}$ [3]. Blue luminescence was reported in some studies due to defects and impurities [4], and the power dependent photoluminescence (PL) of these peaks usually blue shift with increasing power [5]. Although such defects can be destructive in a device, well engineered nanostructure may offer many advantages[6]. Moreover, the smaller the nanostructure, the larger surface-volume ratio and the stronger the surface effects. Combining surface effects and defect engineering will help filling many research gaps. One such research gap is the existing light-emitting diode (LED) phosphor that suffers optical degradation and poor material compatibility. In recent years, much research has been devoted to the development of rare earth doped III-V compound semiconductor based phosphors including GaN-based semiconductors [7, 8]. The GaN and InGaN nanoparticle based phosphors have advantages over other types of phosphors in terms of high quantum efficiency, excitation spectrum well matched to LED emission wavelengths and linewidths, large tunability of excitation and emission wavelength, and high thermal stability.

In this paper, we report on the light emission mechanism of $\mathrm{GaN}$ nanostructures, which showed controllable redshift of $\sim 40 \mathrm{~nm}(\sim 250 \mathrm{meV})$ with increasing optical excitation power. These group-III nitride-based nanostructures were fabricated using UV-assisted electroless chemical etching method. The resultant GaN nanostructures offer good material compatibility with III-V LEDs, high chemical stability, large excitation range, and a life-time compatible to that of the GaN-based LEDs. The nanostructures are simple in fabrication, easy to integrate, and, most importantly, offer tunable broadband emission rendering them a good candidate for tunable color-temperature white LEDs.

\section{Experimental}

The substrate used in this study consisted of a $30 \mu \mathrm{m}$ thick Si-doped GaN epitaxy grown on c-axis (0001) sapphire substrate with a measured resistivity of less than $0.03 \Omega$. $\mathrm{cm}$. The estimated dislocation density and measured carrier concentration of the film are $1 \times 10^{8} \mathrm{~cm}^{-2}$ and $2 \times 10^{18} \mathrm{~cm}^{-3}$, respectively. Prior to etching in a HF: $\mathrm{CH}_{3} \mathrm{OH}: \mathrm{H}_{2} \mathrm{O}_{2}(2: 1: 2)$ solution under $\mathrm{UV}$ illumination, $10 \mathrm{~nm}$ thin stripes of platinum $(\mathrm{Pt})$ were sputtered onto the $\mathrm{GaN}$ samples[9]. The nanostructure layers were later transferred onto a $\mathrm{Si}$ wafer for subsequent room temperature microphotoluminescence $(\mu$-PL) measurements using Jobin Yvon's LabRAM ARAMIS micro-photoluminescence $(\mu P L)$ spectroscopy system. The optical excitation was produced using a Helium-Cadmium (HeCd) laser emitting at $325 \mathrm{~nm}$ with a $<10 \mu \mathrm{m}$ spot-size. The scanning and transmission electron microscopy (SEM and TEM) investigations were performed using FEI Quanta 600 and FEI Titan $\mathrm{G}^{2}$ 80-300 electron microscopes, respectively. 


\section{Results and discussion}

Figure 1 (a) shows the SEM image of the GaN nanostructures sonicated and dispersed on a Si substrate. The nanostructures have the grain-like form with sizes ranging from $10 \mathrm{~nm}$ to $100 \mathrm{~nm}$. Along with these nanostructures we formed nanowires with diameters $\sim 30 \mathrm{~nm}$ and lengths from 1-4 $\mu \mathrm{m}$. Using high resolution TEM (HRTEM), we observed adjoining single-crystal $\mathrm{GaN}$ nanostructures with each particle surrounded by an amorphous-like boundary (Fig. 1(b)). Electron energy loss spectroscopy (EELS) analysis revealed the 20 atomic percentage of oxygen (Fig. $1(\mathrm{c}))$. The presence of oxygen can be explained by the oxidation of $\mathrm{GaN}$ in contact with HF solution producing $\mathrm{Ga}_{2} \mathrm{O}_{3}$.
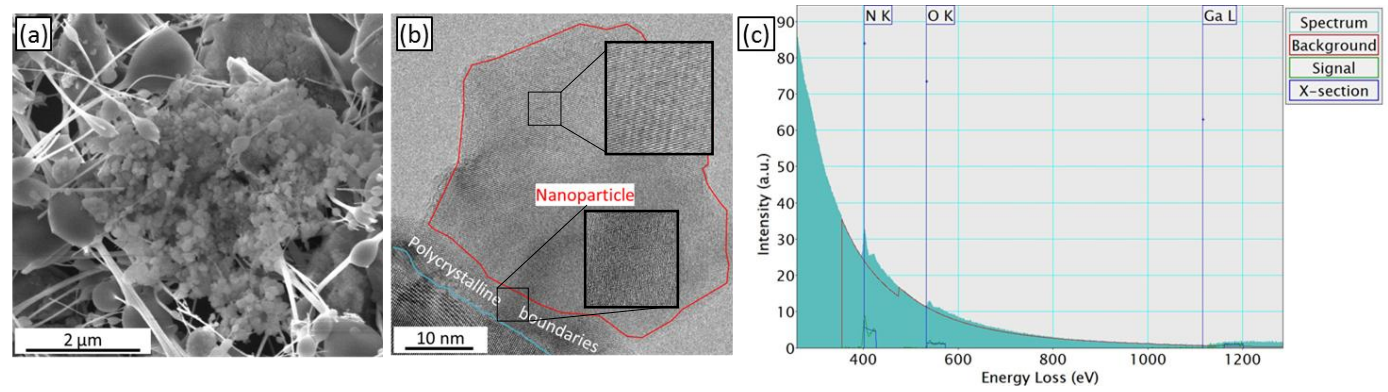

Figure 1. (a) SEM image of the nanostructures prepared using UV metal-assisted electroless etching technique, and (b) TEM image of GaN nanostructure, with the amorphous like boundary (red line) (c) EELS analysis on the GaN nanostructures.

Figure 2 shows the typical room temperature $\mu$-PL emission spectrum of the as-grown $\mathrm{GaN}$ excited at 0.08 $\mathrm{kW} / \mathrm{cm}^{2}$ together with the excitation power dependent $\mu$-PL emission spectra of the GaN nanostructures. For the room temperature PL of the as-grown GaN epitaxy we observe a dominant peat at $3.42 \mathrm{eV}(\sim 362 \mathrm{~nm})$ attributed to the free-exciton to valence band recombination. Most importantly, room temperature PL of GaN nanostructures excited at $0.08 \mathrm{~kW} / \mathrm{cm}^{2}$ exhibits a peak centered at $3.376 \mathrm{eV}(367 \mathrm{~nm})$ which is red-shifted by $34 \mathrm{meV}(5 \mathrm{~nm})$ compared to the as-grown sample. As the excitation power increases from $0.08 \mathrm{~kW} / \mathrm{cm}^{2}$ to $8 \mathrm{~kW} / \mathrm{cm}^{2}$, we observe an almost linear decrease of the peak PL photon energy by $246 \mathrm{meV}$. This was not observed in the as-grown GaN epitaxy with increasing excitation power. Besides the redshift, the measured FWHM increases with the excitation power from $99 \mathrm{meV}(\sim 10 \mathrm{~nm})$ at $0.08 \mathrm{~kW} / \mathrm{cm}^{2}$ to $191 \mathrm{meV}(\sim 24 \mathrm{~nm})$ at $8 \mathrm{~kW} / \mathrm{cm}^{2}$. Such a wide FWHM is twice as large compared to that of the peak from the as-grown $\mathrm{GaN}$, where the line width broadening at the same power density is $42 \mathrm{meV}(\sim 4.5 \mathrm{~nm})$. This FWHM widening indicates a contribution of inhomogeneous broadening in the clusters of nanostructures.

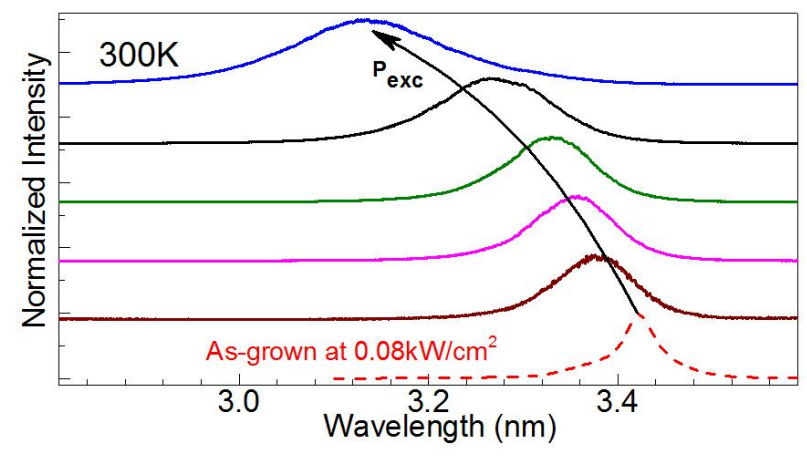

Figure 2. Normalized room temperature $\mu \mathrm{PL}$ emission spectra of as-grown GaN epitaxy (dash line), and GaN nanostructures (solid line) cluster excited with increasing laser power $\left(0.08,0.8,2,4,8 \mathrm{KW} / \mathrm{cm}^{2}\right)$.

In the following discussion, we investigate the large redshift in PL emission in the nanostructures with increasing power density. Due to the extremely high surface to volume ratio of the produced nanostructures, surface states effect is expected to dominate the emission mechanism. The n-type GaN nanostructures have surface defects such as Ga vacancies [10,11], and sidewall oxide formation as evident in the EELS measurement in Fig. 1(c), thus we have band bending in these regions. The creation of surface depletion will change the emission in the GaN nanostructures. The calculated width of the depletion region in our case is $\mathrm{d} \sim 24 \mathrm{~nm}$, given by $d=\sqrt{2 \varepsilon_{G a N} V b i / q N_{d}}$ [12]. Where $\varepsilon_{\mathrm{GaN}}$ is the static dielectric constant of $\mathrm{GaN}, \mathrm{V}_{\mathrm{bi}}$ the potential at the boundary, $\mathrm{q}$ the electronic charge, and $\mathrm{N}_{\mathrm{d}}$ the 
donor density. For nanostructures with a size, $\mathrm{W}<2 \mathrm{~d}$, the nanostructure will be totally depleted. Ga vacancy centers acting like acceptor sites will be depleted from holes, and we then have the free-excitonic (FX) transitions dominating. If $\mathrm{W}>2 \mathrm{~d}$, both depleted region and non-depleted region co-existing. As verified in the excitation power, holes are expected to populate the acceptor-like states in the depletion region and electrons populating the donor-like states, leading to an increase in donor-acceptor pair (DAP) and donor-to-valence band transitions, as the optical and thermal energy excited more carriers to reduce the depletion region, and eventually, visible-emission at a higher excitation power.

\section{Summary}

In summary, we have studied the PL spectrum of GaN nanostructures with size dispersion between $10 \mathrm{~nm}$ to $100 \mathrm{~nm}$ fabricated using the ultraviolet (UV) metal-assisted electroless etching method. A large emission wavelength tunability of $\sim 250 \mathrm{meV}$ has been observed from the nanoparticles. We demonstrated that surface effects are responsible for the redshift dependence of the PL peak with increasing optical power injection. The method used to synthesize these $\mathrm{GaN}$ nanoparticles emitting in the visible is promising for producing III-V semiconductor nanoparticle phosphor.

[1] H. P. T. Nguyen, S. Zhang, K. Cui, X. Han, S. Fathololoumi, M. Couillard, et al., "P-type modulation doped InGaN/GaN dot-in-a-wire whitelight-emitting diodes monolithically grown on $\mathrm{Si}(111), "$ Nano Letters, vol. 11, (2011), pp. 1919-1924.

[2] J. C. Johnson, H. J. Choi, K. P. Knutsen, R. D. Schaller, P. Yang, and R. J. Saykally, "Single gallium nitride nanowire lasers," Nature Materials, vol. 1, (2002), pp. 106-110.

[3] K. M. Song and H. Kim, "Optical Properties of Undoped a-Plane GaN Grown with Different Initial Growth Pressures," Japanese Journal of Applied Physics, vol. 51, (2012), p. 092101.

[4] M. A. Reshchikov and H. Morkoç, "Luminescence properties of defects in GaN," Journal of applied physics, vol. 97, (2005), pp. 061301061301-95.

[5] M. A. Reshchikov, J. Xie, L. He, X. Gu, Y. T. Moon, Y. Fu, et al., "Effect of potential fluctuations on photoluminescence in Mg-doped GaN," phys. stat. sol. (c), vol. 2, (2005), pp. 2761-2764.

[6] A. B. Slimane, A. Najar, T. K. Ng, and B. S. Ooi, "Thermal Annealing induced relaxation of compressive strain in porous GaN structures," (IEEE Photonics Conference, Burlingame, CA, 2012), pp. 921-922.

[7] I. M. Tiginyanu, V. V. Ursaki, L. Sirbu, M. Enaki, and E. Monaico, "Novel phosphors based on porous materials," Physica Status Solidi (C) Current Topics in Solid State Physics, vol. 6, (2009), pp. 1587-1591.

[8] A. J. Steckl, J. Heikenfeld, D. S. Lee, and M. Garter, "Multiple color capability from rare earth-doped gallium nitride," Materials Science and Engineering B: Solid-State Materials for Advanced Technology, vol. 81, (2001), pp. 97-101.

[9] J. A. Bardwell, J. B. Webb, H. Tang, J. Fraser, and S. Moisa, "Ultraviolet photo enhanced wet etching of GaN in K2S2O8 solution," Journal of Applied Physics, vol. 89, (2001), pp. 4142-4149.

[10] M. A. Reshchikov, F. Shahedipour, R. Y. Korotkov, B. W. Wessels, and M. P. Ulmer, "Photoluminescence band near $2.9 \mathrm{eV}$ in undoped GaN epitaxial layers," Journal of Applied Physics, vol. 87, (2000) , pp. 3351-3354.

[11] P. Boguslawski, E. L. Briggs, and J. Bernholc, "Native defects in gallium nitride," Physical Review B, vol. 51, (1995), pp. 17255-17258.

[12] K. Vanheusden, W. L. Warren, C. H. Seager, D. R. Tallant, J. A. Voigt, and B. E. Gnade, "Mechanisms behind green photoluminescence in ZnO phosphor powders," Journal of Applied Physics, vol. 79, (1996) , pp. 7983-7990. 